\title{
Metron: a theoretical journal motivated by real life applications
}

\author{
Giovanni Maria Giorgi ${ }^{1}$
}

Published online: 3 November 2017

C Sapienza Università di Roma 2017

My appointment as Editor in Chief is coming to an end. During my first mandate as Editor in Chief of the Journal (2003-2006), Metron started publishing only articles written in English, therefore changing the habit, due to its founder in 1920, to accept papers written in some of the most widespread languages. During my second appointment (2013-2017) Metron has begun an important collaboration with the international publisher Springer (also thanks to the former EiC and colleague Pier Luigi Conti).

During this period, the editorial line has been to solicit contributions on some aspects of current statistical research taking into account the traditional features of the journal since its foundation. In other words, in agreement with my collaborators, first of all the Executive Editor Marco Alfò to whom my most grateful thanks go, I tried to keep Metron within its traditional field, that is a journal neither in Theoretical nor in Applied Statistics but a journal where theory is motivated by real life applications. Articles that were understood as, essentially, mathematical exercises with a very narrow scope, have been rejected since Statistics is not Mathematics, even though it extensively uses mathematical tools.

After the "International Conference in Memory of Two Eminent Social Scientists: C. Gini and M. O. Lorenz", held in Siena (Italy), May 23-26, 2005, attended by scholars with very different backgrounds, I realized that many of them never had the chance to read, for example, Gini's original work on the famous "concentration ratio". To overcome this and other related inconveniences and make the international scientific community aware of the results achieved by Italian scholars in the early decades of the twentieth century, Metron proposed either complete translations or a selection of excerpts (in the case of very extensive works) of some essential papers. In particular, we may recall the contributions by Gini (1914) on the concentration ratio [translated in Metron 2005, 63(1)], by Pietra (1915) on the Lorenz curve in the continuous case [translated in Metron 2014, 72(1)] and by Gini (1932) on the innovative analytical representation of the concentration curve in a coordinate system which assumes the egalitarian line as $\mathrm{x}$-axis and its perpendicular line as y-axis [Metron 2015, 73(1)]. Some of these results were re-discovered decades later by other scholars. Finally, we

\footnotetext{
Giovanni Maria Giorgi

giovanni.giorgi@uniroma1.it

1 "La Sapienza” University, Rome, Italy
} 
decided to include, in the special issue Transvariation after a hundred years [2017, 75(2)], the translation of the most relevant sections of the paper by Gini (1916) where the concept of transvariation has been introduced.

With the aim to explore some emerging research themes, other special issues have been published during the last few years. We started from "Fuzzy statistical analysis: methods and applications" because of its application in several growing research fields [Metron, 2013, 71(1)] moving through "Advances in Statistical Inference: Bayesian and Likelihood Interplay" where different schools of inference-frequentist and Bayesian-have been compared in the context of complex statistical models [Metron, 2014, 72(2)]. Heterogeneity in the social science context has been investigated in the special issue on "Latent variable models for the analysis of socio-economic data" where models based on either continuous or discrete latent variables have been discussed, with a focus on approaches combining the use of both in the same model [Metron, 2015, 73(2)]. "The Statistical Legacy of Corrado Gini” has provided a thourough analysis of the impact of Corrado Gini on past and present theoretical and applied Statistics for the fiftieth anniversary of his death [Metron, 2016, 74(2)], while, last but not least, "Advances in Survey Statistics" [Metron, 2017, 75(3)] has focused on bringing together valuable and modern contributions in the field of Survey Statistics, with a specific focus on model-based sampling.

I hope I have contributed, together with the entire Editorial Board which deserves my deep and sincere thanks, to enlarge and consolidate this important "publication platform" where theory is strongly motivated by real life applications.

Finally, on behalf of the "Metron family", I extend my sweetest thoughts to two esteemed colleagues and dear friends who recently passed away: Carlo Benedetti (28th February 2015) and Mario Badaloni (28th January 2016). They both have put great efforts as Editors in Chief in order to let our journal be a free forum for meeting and comparing (statistical) ideas.

"La Sapienza" University

Roma, 31st October 2017 